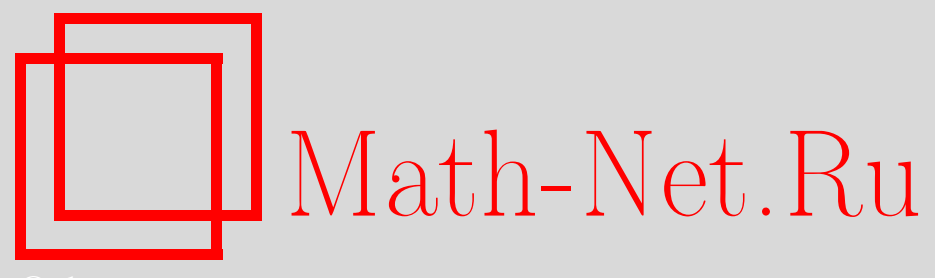

О. Н. Василенко, Об алгоритмах построения изогений эллиптических кривых над конечными полями и их приложениях, Mатем. вопр. криптогр., 2010, том 1, выпуск 1, 7-22

DOI: https://doi.org/10.4213/mvk2

Использование Общероссийского математического портала Math-Net.Ru подразумевает, что вы прочитали и согласны с пользовательским соглашением http://www.mathnet.ru/rus/agreement

Параметры загрузки:

IP: 18.234 .156 .22

26 апреля 2023 г., 09:46:51

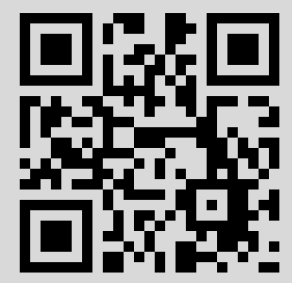




\title{
Об алгоритмах построения изогений ЭЛЛиптических кривых над конечными полями и их приложениях
}

\author{
О. Н. Василенко \\ Лаборатория ТВП, Москва
}

Получено 22.VI.2010

В работе описан алгоритм Галбрайта построения изогений эллиптических кривых над конечными полями и несколько его модификаций. Обсуждаются применения изогенных элиптических кривых над конечными полями.

Ключевые слова: изогении.

On, algorithmic constructions of isogenies of elliptic curves over finite fields and their applications

\section{O. N. Vasilenko \\ TVP Laboratory, Moscow}

\begin{abstract}
An algorithm of S. D. Galbraith for constructing isogenies of elliptic curves over finite fields are described together with some its modifications. Applications of isogenies of elliptic curves over finite fields are discussed.
\end{abstract}

Key words: curves over finite fields, isogenies.

Citation: Mathematical Aspects of Cryptography, 2010, vol. 1, no. 1, pp. 7-22 (Russian). 


\section{1. Введение}

Пусть $E_{1}, E_{2}$ - эллиптические кривые над конечным полем $\mathrm{GF}(q)$. Изогенией кривых называется рациональное отображение, переводящее ноль в ноль. Теорема Тейта (см. [25]) гласит, что $E_{1}$ и $E_{2}$ изогенны над $\mathrm{GF}(q)$ тогда и только тогда, когда порядки групп точек $\left|E_{i}(\mathrm{GF}(q))\right|$ совпадают. Поскольку эти порядки можно вычислить с полиномиальной сложностью с помощью алгоритмов Шуфа - Элкиса - Аткина, метода циклов изогений, методов Сато Фуке-Годри - Хэрли (см. [2, гл. 4; 27, гл. 4]), мы можем достаточно быстро определить, изогенны ли данные кривые. В 1999 году С.Галбрайт [13], основываясь на результатах диссертации Кохеля [19], предложил достаточно эффективный во многих случаях алгоритм построения изогении кривых $E_{1}, E_{2}$. Этот алгоритм нашел несколько интересных практических приложений, в частности в задаче дискретного логарифмирования в группе точек эллиптической кривой над конечным простым полем.

В данной работе мы опишем алгоритм Галбрайта и некоторые его модификации, а также некоторые приложения изогений эллиптических кривых над конечными полями.

Необходимые определения и сведения из теории эллиптических кривых можно найти в книге [23].

Пусть $E$ - эллиптическая кривая над конечным полем $\mathrm{GF}(q)$ характеристики $p>3$, задаваемая уравнением

$$
y^{2}=x^{3}+a x+b
$$

Через $E(\mathrm{~F})$ мы обозначаем группу точек эллиптической кривой с координатами в поле $\mathrm{F} \supseteq \mathrm{GF}(q)$, включая бесконечно удаленную точку, а через $E(\mathrm{~F})[l]-$ группу точек, имеющих порядок $l$. Пусть $\phi-$ отображение Фробениуса, $\phi((x, y))=\left(x^{q}, y^{q}\right)$, удовлетворяющее уравнению

$$
X^{2}-t X+q=0
$$

где $t$ - след отображения Фробениуса, $|t| \leq 2 \sqrt{q},|E(\mathrm{GF}(q))|=q+1-t$. Пусть $\operatorname{End}(E)$ - кольцо эндоморфизмов кривой, являющееся порядком кольца $\mathbb{Z}_{\mathbb{K}}$,

$$
\mathbb{Z}_{\mathbb{K}} \supseteq \operatorname{End}(E) \supseteq \mathbb{Z}[\phi]
$$

где $\mathbb{Z}_{\mathbb{K}}$ - кольцо целых алгебраических чисел мнимого квадратичного поля $\mathbb{K}=\mathbb{Q}(\sqrt{d}), \quad d=t^{2}-4 q<0$.

Кондуктором порядка $\mathcal{O}$ в $\mathbb{Z}_{\mathbb{K}}$ мы будем называть величину $c=c(\mathcal{O})=$ $=\left[\mathbb{Z}_{\mathbb{K}}: \mathcal{O}\right]$. Эту величину можно вычислить, зная базис $\mathcal{O}$ над $\mathbb{Z}$. Действитель- 
но, пусть $d=d_{1}^{2} d_{2}$, где $d_{2}$ бесквадратно. Тогда $\mathbb{K}=\mathbb{Q}\left(\sqrt{d_{2}}\right)$, и целый базис $\mathbb{K}$ хорошо известен (см., например, [24]). Кондуктор порядка будет равен модулю определителя матрицы перехода от базиса $\mathbb{Z}_{\mathbb{K}}$ к базису $\mathcal{O}$. Заметим, что для вычисления кондуктора нам необходимо найти факторизацию $d$.

Кондуктором эллиптической кривой мы будем называть кондуктор порядка $\mathbb{Z}[\phi]$. Через $j(E)$ мы будем обозначать $j$-инвариант кривой.

Пусть $\varphi: E_{1} \longrightarrow E_{2}-$ изогения простой степени $l$ эллиптических кривых $E_{1}, E_{2}$, определенных над полем $\mathrm{GF}(q)$, и таких, что кольца их эндоморфизмов равны $\mathbb{Z}_{\mathbb{K}}$. Идеалом ядра изогении называется идеал

$$
I(\operatorname{Ker}(\varphi))=\left\{\psi \in \operatorname{End}\left(E_{1}\right) \mid \psi(\operatorname{Ker}(\varphi))=0\right\} .
$$

Это идеал кольца $\mathbb{Z}_{\mathbb{K}}$ нормы $l$.

Пусть $\varphi: E_{1} \longrightarrow E_{2}-$ изогения степени $m=\operatorname{deg} \varphi$ эллиптических кривых $E_{1}, E_{2}$, определенных над полем $\mathrm{GF}(q)$. Через $\hat{\varphi}$ мы будем обозначать дуальную изогению, $\hat{\varphi} \circ \varphi=[\mathrm{m}]_{1}, \varphi \circ \hat{\varphi}=[\mathrm{m}]_{2}$, где $[\mathrm{m}]_{1},[\mathrm{~m}]_{2}-$ отображения умножения на $m$ на кривых $E_{1}$ и $E_{2}$ соответственно.

\section{2. Алгоритм Галбрайта: построение изогений над про- стым полем}

В этом параграфе мы будем рассматривать ординарные кривые $E_{1}, E_{2}$ над простым полем характеристики $p>3$. Мы предполагаем, что известен порядок групп точек кривых

$$
p+1-t=\left|E_{1}(\mathrm{GF}(p))\right|=\left|E_{2}(\mathrm{GF}(p))\right| .
$$

Отображение Фробениуса будет иметь вид $\phi((x, y))=\left(x^{p}, y^{p}\right)$. Будем считать, что кондуктор кривых $c$ уже вычислен.

Фиксируем мнимое квадратичное поле $\mathbb{K}=\mathbb{Q}(\sqrt{d}), d=t^{2}-4 p<0$, и обозначим через $D$ дискриминант поля $\mathbb{K}$.

Опишем алгоритм Галбрайта [13] для построения изогении $E_{1}$ и $E_{2}$. Построение этой изогении сводится к построению цепочки изогений простых степеней $l, l \neq p$. В основе метода лежит следующая теорема.

Теорема 1 (см. [19]). Пусть $\varphi: E_{1}^{\prime} \longrightarrow E_{2}^{\prime}-$ изогения простой степени $l \neq p$ ординарных эллиптических кривых $E_{1}^{\prime}, E_{2}^{\prime}$, определенных над полем $\mathrm{GF}(p), p+1-t=\left|E_{1}^{\prime}(\mathrm{GF}(p))\right|=\left|E_{2}^{\prime}(\mathrm{GF}(p))\right|$. Обозначим через $\mathcal{O}_{i}=\operatorname{End}\left(E_{i}^{\prime}\right), i=1,2$. Тогда либо $\mathcal{O}_{1} \supseteq \mathcal{O}_{2}$, либо $\mathcal{O}_{2} \supseteq \mathcal{O}_{1}$ в кольие $\mathbb{Z}_{\mathbb{K}}$. 
При этом l делится на индекс одного порядка в другом и делит кондуктор кривой (для $E_{1}^{\prime}$ и $E_{2}^{\prime}$ этот кондуктор и числовое поле $\mathbb{K}=\mathbb{Q}(\sqrt{d})$ совпадают).

Определение. Пусть $\varphi: E_{1}^{\prime} \longrightarrow E_{2}^{\prime}-$ изогения простой степени $l \neq p$ ординарных эллиптических кривых $E_{1}^{\prime}, E_{2}^{\prime}$. Если $\mathcal{O}_{i}=\operatorname{End}\left(E_{i}^{\prime}\right), i=1,2$, совпадают, то $\varphi$ называется «горизонтальной» в $l$. Если $\mathcal{O}_{1} \supseteq \mathcal{O}_{2}$ с индексом $l$, то $\varphi$ называется изогенией «вниз» в $l$. Если $\mathcal{O}_{2} \supseteq \mathcal{O}_{1}$ с индексом $l$, то $\varphi$ называется изогенией «вверх» в $l$. Если $l$ не делит индекс $\left[\mathbb{Z}_{\mathbb{K}}: \operatorname{End}\left(E_{1}^{\prime}\right)\right]$, то невозможно расширить кольцо эндоморфизмов в $l$ раз, и тогда говорят, что $E_{1}^{\prime}$ находится «наверху» в $l$. Если $l$ не делит индекс $\left[\operatorname{End}\left(E_{1}^{\prime}\right): \mathbb{Z}[\phi]\right]$, то невозможно сузить кольцо эндоморфизмов в $l$ раз, и тогда говорят, что $E_{1}^{\prime}$ находится «внизу»в $l$.

Пусть $\mathcal{L}=\left\{l \mid l-\right.$ простое, $\left.\left(\frac{D}{l}\right) \in\{0,1\}, l<6(\ln |D|)^{2}\right\}$.

Напомним, что по $j$-инварианту эллиптическая кривая восстанавливается с точностью до изоморфизма. Кроме того, хорошо известно, что если две заданные кривые связаны изогенией данной простой степени $l$, то сама изогения строится по формулам Велу и Элкиса (см. [9; 28], а также [3; 7; 13; $19 ; 20 ; 22])$. Мы не будем приводить эти формулы, поскольку в одном из следующих параграфов опишем более эффективный для решения этой задачи метод работы [5].

\section{Алгоритм Галбрайта}

1 этап. Вычислить классические модулярные полиномы $\Phi_{l}(x, y) \in$ $\mathrm{GF}(p)[x, y]$ для каждого простого $l \mid c$ и каждого $l \in \mathcal{L}$. (Определение и стандартный способ вычисления см., например, в $[10 ; 3$, гл. $3 ; 6$, гл.7; 20].)

2 этап. Для $i=1,2$ построить цепь изогений из $E_{i}$ в некоторую эллиптическую кривую $E_{i}^{\prime}$, у которой $\operatorname{End}\left(E_{i}^{\prime}\right)=\mathbb{Z}_{\mathbb{K}}$. Для этого надо выполнить следующие действия.

Фиксируем $E-$ одну из кривых $E_{1}, E_{2}$. Для каждого простого $l \mid c$ обозначим $l^{b(l)}$ степень $l$ в разложении $c$ на простые множители. Фиксируем такое $l$. Сейчас мы найдем степень $l$ в кондукторе $\operatorname{End}(E)$. Находим корни $\Phi_{l}(j(E), y)(\bmod p)$. Если корень только один, то можно доказать (см. [13; $19])$, что $E$ находится «внизу» в $l$ и кондуктор $\operatorname{End}(E)$ делится ровно на $l^{b(l)}$. В противном случае $\Phi_{l}(j(E), y)(\bmod p)$ раскладывается на линейные множители. Если $b(l)=1$, то $l$ не делит кондуктор End $(E)$. Если же $b(l)>1$, то возьмем один из корней $\Phi_{l}(j(E), y)(\bmod p)-$ пусть это $j_{0}$. Мы можем определить, что это $j$-инвариант кривой, находящейся «внизу», рассматривая корни $\Phi_{l}\left(j_{0}, y\right) \bmod p$. Если мы еще не «внизу», то берем один из корней и с ним поступаем так же, и т.д. Если мы сделаем $b^{\prime}(l)$ шагов, пока не достигнем «низа», то кондуктор $\operatorname{End}(E)$ делится ровно на $l^{b(l)-b^{\prime}(l)}$. 
Теперь, зная степень $l$, делящую кондуктор $\operatorname{End}(E)$, мы точно так же можем идти «вверх», пока не достигнем кривой $E^{\prime}$, находящейся «наверху» в $l$. Именно, если кондуктор делится на $l^{b(l)}$, то мы уже «наверху». Иначе мы рассматриваем корни $\Phi_{l}(j(E), y)(\bmod p)$. Нам надо найти среди них такое $j^{\prime}$, что $\Phi_{l}\left(j^{\prime}, y\right)(\bmod p)$ раскладывается на линейные множители. Т. е. мы (пользуясь симметричностью классических модулярных полиномов) для корней $x^{\prime}$ уравнения $\Phi_{l}(j(E), y) \equiv 0(\bmod p)$ проверяем, что $\Phi_{l}\left(x^{\prime}, y\right)(\bmod p)$ раскладывается на линейные множители. Найдя такой корень (являющийся $j$-инвариантом кривой «над» нашей), мы затем аналогично поступаем с ним и т. д., пока не окажемся «наверху».

Теперь мы берем эту находящуюся «наверху» кривую, берем другое простое число, делящее кондуктор кривой, и продолжаем этот процесс. В конце мы найдем кривую $E^{\prime}$, «над» которой нет кривых, т. е. ее кольцо эндоморфизмов равно $\mathbb{Z}_{\mathbb{K}}$. В итоге мы нашли цепочку изогений простой степени, соединяющих $E$ и $E^{\prime}$.

Мы обозначаем эти построенные кривые для $E_{1}, E_{2}$ через $E_{1}^{\prime}, E_{2}^{\prime}$ соответственно. Через $j_{1}, j_{2}$ мы обозначаем $j$-инварианты построенных кривых $E_{i}^{\prime}$.

3 этап. На этом этапе мы соединим цепью изогений кривые $E_{1}^{\prime}, E_{2}^{\prime}$.

Мы выращиваем два дерева изогений, начиная с двух вершин, помеченных $j_{1}, j_{2}$. На очередном шаге мы выбираем одно из двух деревьев, выбираем случайное $l \in \mathcal{L}$ и для каждой вершины $j$ в выбранном дереве находим корни $\Phi_{l}(j, y)(\bmod p)$. Для каждого из найденных корней, в случае отсутствия в обоих деревьях вершины, помеченной этим корнем, мы добавляем такую вершину в выбранное дерево и соединяем ее с вершиной $j$. Мы повторяем такие шаги до тех пор, пока не появится ребро, соединяющее два дерева.

Таким образом, на этом этапе мы построим цепочку изогений простой степени, соединяющих $E_{1}^{\prime}, E_{2}^{\prime}$.

4 этап. Пользуясь цепочками изогений, построенных на этапах 2 и 3 , находим цепочку изогений простых степеней от $E_{1}$ к $E_{2}$. Для каждой из них мы находим явные формулы (например, методом Велу-Элкиса). Рекомендуется хранить эти изогении по отдельности, не вычисляя их композицию. Так как мы вычисляли промежуточные кривые по их $j$-инвариантам, то в конце цепочки получится кривая $E_{2}^{\prime \prime}$, изоморфная $E_{2}$.

5 этап. С помощью известных формул (см., например, [3; 13]) вычисляем изоморфизм $E_{2}^{\prime \prime}$ и $E_{2}$. Этим завершается работа алгоритма. Искомая изогения будет композицией построенных выше. 
Теорема 2 (см. [13]). При условии справедливости гипотезы Римана для дзета-функиий Дедекинда мнимых квадратичных полей средняя оценка сложности описанного выме алгоритма составляет $O\left(p^{3 / 2} \ln p\right)$ операций поля $\mathrm{GF}(p)$. Для его работы требуется пространство памяти величиной $O(p \ln p)$ битов.

ЗАмечАНИЕ 1. В ряде случаев алгоритм работает быстрее, см. [13]. Например, если кондуктор является $O(\ln p)$-гладким, то средняя сложность составляет $O\left(p^{1 / 4}(\ln p)^{13 / 2}\right)$ операций поля $\mathrm{GF}(p)$ (однако для получения такой оценки необходимы некоторые эвристические допущения). Если же число классов идеалов поля $\mathbb{K}$ мало (порядка $O\left((\ln p)^{\text {const }}\right)$ ), то алгоритм может даже иметь полиномиальную сложность.

ЗАМЕчАНИЕ 2. Описанный выше алгоритм непрактичен, когда кондуктор кривой делится на большое простое число.

\section{3. Построение изогений над произвольным конечным полем}

Описанный выше алгоритм Галбрайта применим к кривым над простыми полями характеристики $p>3$. В работе [14] предложен алгоритм построения изогений, применимый для кривых над любыми конечными полями. В нем используются вычисления с дробными идеалами числового поля (идеалами ядер изогений). Ниже мы вкратце опишем его схему.

Мы предполагаем, что заданы две ординарные эллиптические кривые $E_{1}, E_{2}$ над конечным полем $\mathrm{GF}(q)$ характеристики $p \geq 2$. Мы считаем их изогенными над этим полем, что определяется путем вычисления порядков групп точек. Пусть $\phi-$ отображение Фробениуса и $t-$ его след. Пусть $\operatorname{End}\left(E_{1}\right)$, End $\left(E_{2}\right)$ - кольца эндоморфизмов кривых, являющиеся порядками кольца $\mathbb{Z}_{\mathbb{K}}$ целых алгебраических чисел мнимого квадратичного поля $\mathbb{K}=\mathbb{Q}(\sqrt{d}), d=t^{2}-4 q<0$.

Алгоритм построения изогении $\varphi: E_{1} \longrightarrow E_{2}$ состоит из следующих стадий.

1 стадия. Сведение к случаю изогении кривых $E_{1}^{\prime}, E_{2}^{\prime}$, у которых кольца эндоморфизмов являются максимальными порядками. Эта стадия выполняется аналогично описанному выше алгоритму Галбрайта.

2 стадия. На этой стадии мы определяем цепочку идеалов в $\mathbb{Z}_{\mathbb{K}}$, соответствующих искомой изогении $E_{1}^{\prime}, E_{2}^{\prime}$. Здесь используется техника вычислений, аналогичная алгоритмам Шуфа - Элкиса - Аткина (см. [3, гл. 7]).

Пусть $j$ есть $j$-инвариант эллиптической кривой $E$, у которой кольцо эндоморфизмов является максимальным порядком поля $\mathbb{K}$. Пусть $l-$ простое 
число, и мы хотим построить изогению из нашей кривой, имеющую степень $l$. Такая изогения соответствует некоторому простому идеалу поля $\mathbb{K}$. Это соответствие устанавливается следующим образом. Предположим, что идеал $(l)$ расщепляется в $\mathbb{Z}_{\mathbb{K}}$ (другие случаи рассматриваются аналогично). Тогда

$$
X^{2}-t X+q \equiv(X-\mu)(X-\lambda) \quad(\bmod l)
$$

$\mu, \lambda \in \mathbb{Z}$, и $(l)=\mathfrak{l}_{1} \mathfrak{l}_{2}$, где $\mathfrak{l}_{1}=(l, \phi-\mu), \mathfrak{l}_{2}=(l, \phi-\lambda)$ - простые идеалы $\mathbb{Z}_{\mathbb{K}}$. В этом случае модулярный полином $\Phi_{l}(j, X)$ имеет два корня $j_{1}, j_{2}$ в $\mathrm{GF}(q)$, являющихся $j$-инвариантами двух изогенных нашей кривой, причем степени изогений из $E$ в эти кривые равны $l$, а идеалы ядер $-\mathfrak{l}_{1}, \mathfrak{l}_{2}$. Чтобы определить, какой из идеалов соответствует какому из $j$-инвариантов, мы находим подгруппу $C_{1}$ в $E[l]$, лежащую в ядре изогении из $E$ в кривую $E^{\prime \prime}$ с $j$-инвариантом $j_{1}$, а затем для какой-либо ненулевой точки $P$ в этой подгруппе проверяем равенство $\phi(P)=\mu P$. Если оно выполнено, то $j_{1}$ соответствует $\mathfrak{l}_{1}$, иначе $\mathfrak{l}_{2}$ (аналогичные вычисления использовались ранее в алгоритме Элкиса для вычисления порядка группы точек эллиптической кривой).

Мы теперь рассмотрим некоторое случайное блуждание по множеству пар $(j, \mathfrak{a})$, где $j$ есть $j$-инвариант некоторой кривой $E$, а $\mathfrak{a}$ - некоторый идеал $\mathbb{Z}_{\mathbb{K}}$. Шаги этого блуждания соответствуют изогениям небольшой простой степени $l$, причем при заданных $l,(j, \mathfrak{a})$ следующая пара есть $\left(j_{1}, \mathfrak{b}\right)$, где $j_{1}-$ $j$-инвариант изогенной кривой с идеалом ядра $\mathfrak{l}_{1}$ (см. выше), a $\mathfrak{b}=\mathfrak{a l}_{1}$ (идеалы мы храним с помощью эрмитовой нормальной формы, см. [6, гл. 4]). Если множество рассматриваемых простых чисел $l$ выбрано правильно (теоретически надо рассматривать все $l \leq 6(\log D)^{2}$, где $D-$ дискриминант числового поля), то после некоторого не очень большого числа шагов будет найдена коллизия $j_{1}^{\left(s_{1}\right)}=j_{2}^{\left(s_{2}\right)}$ пар $\left(j_{1}^{\left(s_{1}\right)}, \mathfrak{a}^{\left(s_{1}\right)}\right),\left(j_{2}^{\left(s_{2}\right)}, \mathfrak{a}^{\left(s_{2}\right)}\right)$. Искомая изогения из $E_{1}^{\prime}$ в $E_{2}^{\prime}$ будет соответствовать идеалу $\mathfrak{a}_{0}=\mathfrak{a}^{\left(s_{1}\right)} / \mathfrak{a}^{\left(s_{2}\right)}$.

Мы видим, что искомой изогении соответствует идеал $\mathfrak{a}_{0}$, равный произведению большого (теоретически экспоненциального) числа простых идеалов небольшой простой нормы.

3 стадия. На этой стадии мы сглаживаем построенный идеал $\mathfrak{a}_{0}$. Для этого мы рассматриваем множество простых идеалов $\mathcal{F}^{\prime}$ кольца $\mathbb{Z}_{\mathbb{K}}$, соответствующих простым числам $l \leq(\log q)^{2}$. Используя эрмитовы нормальные формы для представления целых идеалов, ищем случайный набор целых чисел $\left\{a_{\mathfrak{l}}\right\}$, для которого идеал $\mathfrak{a}_{0} \prod_{\mathfrak{l} \in \mathcal{F}^{\prime}} \mathfrak{l}^{a_{\mathfrak{l}}}$ разложится в произведение простых идеалов $\mathcal{F}^{\prime}$ :

$$
\mathfrak{a}_{0} \prod_{\mathfrak{l} \in \mathcal{F}^{\prime}} \mathfrak{l}^{a_{\mathfrak{l}}}=\prod_{\mathfrak{l} \in \mathcal{F}^{\prime}} \mathfrak{l}^{b_{\mathfrak{l}}} .
$$


Отсюда

$$
\mathfrak{a}_{0}=\prod_{\mathfrak{l} \in \mathcal{F}^{\prime}} \mathfrak{l}^{b_{\mathfrak{l}}-a_{\mathfrak{l}}} .
$$

В этом представлении количество сомножителей не очень велико, что позволит на следующем этапе построить искомую изогению достаточно быстро.

4 стадия. Строим изогению $E_{1}^{\prime}, E_{2}^{\prime}$. Для этого по очереди берем каждый простой идеал в (1), с помощью описанного выше метода находим соответствующий ему $j$-инвариант очередной изогенной кривой и строим очередную изогению в эту кривую. В итоге будет построена искомая цепочка изогений от $E_{1}^{\prime}$ к $E_{2}^{\prime}$ (явные формулы для них находятся по формулам Велу - Элкиса).

Конец описания схемы алгоритма.

Теорема 3 (см. [14]). Оиенка сложности описанного выше алгоритма составляет в худшем случае не более $O\left(q^{3 / 2+\epsilon}\right)$ операций поля $\mathrm{GF}(q)$. Для его работы требуется пространство памяти величиной $O\left(q^{1+\epsilon}\right)$ битов. Средняя оценка сложности составляет $O\left(q^{\frac{1}{4}+\epsilon}\right)$ операций.

\section{4. О построении изогений простой степени}

Пусть заданы ординарные кривые $E_{1}, E_{2}$ над полем $\mathrm{GF}(q)$ характеристики $p>3$, и нам известно, что существует изогения $\varphi$ из $E_{1}$ в $E_{2}$ простой степени $l$, отличной от характеристики поля. Такая изогения имеет вид

$$
\varphi(x, y)=\left(\frac{\varphi_{1}(x, y)}{\psi(x)^{2}}, \frac{\varphi_{2}(x, y)}{\psi(x)^{3}}\right)
$$

(см. [9; 19]), где

$$
\psi(x)=\prod_{\left(x_{0}, \pm y_{0}\right) \in \operatorname{Ker}(\varphi)}\left(x-x_{0}\right),
$$

$\varphi_{1}, \varphi_{2}$ - многочлены. Многочлен $\psi(x)$ вычисляется с помощью метода Элкиса (см. [9]), а затем с его помощью по формулам Велу (см. [28]) находятся $\varphi_{1}, \varphi_{2}$ (см. также [10]).

В работе [5] был предложен более эффективный способ, имеющий лучшую оценку сложности. Ниже мы опишем его.

Мы предполагаем, что кривые заданы уравнениями

$$
y^{2}=x^{3}+A_{i} x+B_{i}, \quad i=1,2 .
$$


Согласно [5, Предложение 4.1], искомая изогения кривых может быть представлена в виде

$$
\varphi(x, y)=\left(N(x) / D(x), y(N(x) / D(x))^{\prime}\right)
$$

где

$$
D(x)=\prod_{\left(x_{0}, y_{0}\right) \in \operatorname{Ker}(\varphi)^{*}}\left(x-x_{0}\right)=x^{l-1}-\sigma_{2} x^{l-2}+\sigma_{3} x^{l-3}-\sigma_{4} x^{l-4}+\ldots,
$$

а многочлен $N(x)$ связан с $D(x)$ формулой

$N(x) / D(x)=l x-\sigma_{2}-\left(3 x^{2}+A_{1}\right) D^{\prime}(x) / D(x)-2\left(x^{3}+A_{1} x+B_{1}\right)\left(D^{\prime}(x) / D(x)\right)^{\prime}$.

Далее мы будем работать с формальными степенными рядами

$$
f=\sum_{i} f_{i} x^{i}, \quad f_{i} \in \mathrm{GF}(q),
$$

имеющими лишь конечное число отрицательных степеней. Множество таких рядов мы обозначаем $\mathrm{GF}(q)[[x]]$.

Нетрудно показать (см. [5]), что

$$
N(x) / D(x)=1 / S(1 / \sqrt{x})^{2},
$$

где формальный степенной ряд $S(x)$ имеет вид

$$
S(x)=x+\frac{A_{2}-A_{1}}{10} x^{5}+\frac{B_{2}-B_{1}}{14} x^{7}+O\left(x^{9}\right) \in x+x^{3} \mathrm{GF}(q)\left[\left[x^{2}\right]\right]
$$

и удовлетворяет дифференциальному уравнению

$$
\left(B_{1} x^{6}+A_{1} x^{4}+1\right) S^{\prime}(x)^{2}=1+A_{2} S(x)^{4}+B_{2} S(x)^{6} .
$$

Мы будем обозначать через $T(x)$ такой ряд, для которого $S(x)=$ $=x T\left(x^{2}\right)$.

\section{Алгоритм построения изогении}

1 шаг. Вычислить многочлен

$$
C(x)=\left(B_{1} x^{6}+A_{1} x^{4}+1\right)^{-1} \quad\left(\bmod x^{8 l-3}\right) \in \mathrm{GF}[[x]] .
$$


2 шаг. Вычислить многочлен $S(x)\left(\bmod x^{8 l-3}\right)$, пользуясь результатами шага 1 и используя для последовательного определения коэффициентов дифференциальное уравнение (2). Затем определить коэффициенты многочлена $T(x)\left(\bmod x^{4 l-2}\right)$, пользуясь равенством

$$
S(x) \equiv x T\left(x^{2}\right) \quad\left(\bmod x^{8 l-3}\right) .
$$

3 шаг. Вычислить

$$
U(x) \equiv 1 / T(x)^{2} \quad\left(\bmod x^{4 l-2}\right) .
$$

Отсюда, пользуясь стандартной техникой, восстановить коэффициенты рациональной функции $U(x)=1 / T(x)^{2}$.

4 шаг. Выдать

$$
N(x) / D(x)=x U(1 / x) .
$$

Конец описания алгоритма.

Обоснование корректности см. в [5]. Оценка сложности указанного метода составляет $O(M(l) \log l)$ операций поля $\mathrm{GF}(q)$, где $M(n)$ обозначает количество операций поля, необходимых для умножения двух многочленов степени не выше $n$.

\section{5. Использование изогений в задаче дискретного ло- гарифмирования на эллиптических кривых над ко- нечными полями}

В этом параграфе мы рассмотрим возможность применения изогений в алгоритмах дискретного логарифмирования на эллиптических кривых над конечными полями, основанных на спуске Вейля, см. [16; 4, гл. 8; 17, гл. 4].

Мы ограничимся случаем, когда эллиптическая кривая $E$, заданная над конечным полем $\mathrm{K}=\mathrm{GF}\left(2^{m}\right)$, является ординарной. Предположим, что $|E(\mathrm{~K})|=n h$, где $n-$ простое, а $h-$ невелико. В 1998 году Фрей [12] предложил использовать спуск Вейля для сведения задачи дискретного логарифмирования в группе $E(\mathrm{~K})$ к задаче дискретного логарифмирования на якобиане кривой рода больше 1 , однако определенной над меньшим полем $\mathrm{k}=\mathrm{GF}\left(2^{l}\right)$, где $m=l d$. Точнее, рассматривается ограничение Вейля $\operatorname{Res}_{\mathrm{K} / \mathrm{k}}(E)$, являющееся абелевым многообразием размерности $d$ над $\mathrm{k}$, причем группа $\operatorname{Res}_{\mathrm{K} / \mathrm{k}}(E)(\mathrm{k})$ его k-рациональных точек изоморфна группе $E(\mathrm{~K})$, и следовательно, задачи дискретного логарифмирования в этих 
группах эквивалентны (изоморфизм вычисляется достаточно просто, см. [12; 16]). Затем в методе Фрея делаются попытки построить кривую $\mathcal{C}$, определенную над $\mathrm{k}$ и содержащуюся в $\operatorname{Res}_{\mathrm{K} / \mathrm{k}}(E)$, такую, что на якобиане $J_{\mathcal{C}}$ этой кривой существует алгоритм дискретного логарифмирования, более быстрый, чем $\rho$-метод Полларда, и при этом уравнение дискретного логарифмирования в группе $E(\mathrm{~K})$ может быть эффективно отображено в уравнение дискретного логарифмирования в $J_{\mathcal{C}}$. Идеи Фрея получили развитие в работе [15].

В 2002 г. Годри, Хесс и Смарт в работе [16] показали, как пересечь ограничение Вейля $\operatorname{Res}_{\mathrm{K} / \mathrm{k}}(E)$ с $n$ гиперплоскостями для того, чтобы получить искомую гиперэллиптическую кривую $\mathcal{C}$ некоторого рода $g$, и предложили эффективный (в большинстве случаев) алгоритм, сводящий дискретное логарифмирование в группе $E(\mathrm{~K})$ к задаче дискретного логарифмирования на якобиане $J_{\mathcal{C}}$ этой кривой. В свою очередь, для решения этой последней задачи существует использующий факторные базы алгоритм Энге-Годри (см. [11]) со средней оценкой сложности $\exp \left\{(\sqrt{2}+o(1)) \sqrt{g \log 2^{l} \log \left(g \log 2^{l}\right)}\right\}$ битовых операций (при $g / l \longrightarrow \infty$ ). Таким образом, если род кривой $g$ не очень велик, то мы получаем субэкспоненциальный алгоритм для решения задачи дискретного логарифмирования на исходной эллиптической кривой. Данный подход сокращенно называется атакой GHS.

Позднее (см. для справок [17, гл. 4]) было показано, что атака GHS является неэффективной для всех представляющих практический интерес эллиптических кривых над полями $\mathrm{GF}\left(2^{m}\right)$ для всех простых $m, 160 \leq m \leq 600$. Точнее, было показано, что род получающихся кривых либо слишком мал, либо слишком велик, чтобы с их помощью эффективно получать информацию об искомом дискретном логарифме.

Однако было показано, что для некоторых эллиптических кривых и некоторых составных значений $m, 160 \leq m \leq 600$, атака GHS является эффективной. Хотя доля таких кривых невелика, мы можем эффективно распространить эту атаку на большее количество кривых, используя изогении. Рассмотрим этот вопрос более детально.

Пусть заданы две эллиптические кривые $E_{1}, E_{2}$, заданные над конечным полем $\mathrm{K}$ и изогенные над этим полем. Тогда изогения $\varphi: E_{1} \longrightarrow E_{2}$ является гомоморфизмом групп $E_{1}[\mathrm{~K}], E_{2}[\mathrm{~K}]$ и задача дискретного логарифмирования $P=\lambda Q$ в группе $E_{1}[\mathrm{~K}]$ преобразуется в задачу дискретного логарифмирования $\varphi(P)=\lambda \varphi(Q)$ в группе $E_{2}[\mathrm{~K}]$ (обратный переход осуществляется с помощью дуальной изогении $\hat{\varphi}$ ). При этом может оказаться, что задача дискретного логарифмирования на кривой $E_{2}$ эффективно решается с помощью атаки GHS, в то время как на кривой $E_{1}$ атака GHS неэффективна. Ес- 
ли кривая $E_{1}$ задана, то для нахождения кривой $E_{2}$, на которой атака GHS эффективна, возможны два подхода:

1) перебирая кривые $E$, для которых атака GHS эффективна, проверять, что $\left|E_{1}[\mathrm{~K}]\right|=|E[\mathrm{~K}]|$, и если равенство выполнено, то построить изогению $\varphi: E_{1} \longrightarrow E$ с помощью одного из описанных выше алгоритмов;

2) перебирая случайным образом кривые $E$, для которых существует изогения $\varphi: E_{1} \longrightarrow E$, проверять, будет ли для них атака GHS эффективной.

В $[4$, гл. 8$]$ получены некоторые оценки для вероятности реализации первого подхода.

Теорема 4. Пусть $E, E_{1}, \ldots, E_{N}$ над полем $G F\left(2^{m}\right)$ выбраны случайно, равновероятно и независимо. Тогда вероятность того, что Е изогенна одной из кривых $E_{1}, \ldots, E_{N}$, не меньше, чем

$$
1-\left(1-\frac{1}{2^{m / 2+1}+2}\right)^{N}
$$

Отсюда, в частности, следует, что если количество кривых $N$, для которых атака GHS эффективна, достаточно велико, то вероятность успешной реализации первого подхода также может быть достаточно большой.

Некоторые результаты о связи задач дискретного логарифмирования на изогенных кривых получены также в работе [18].

\section{6. Некоторые другие приложения изогений}

Здесь мы рассмотрим возможность использования изогений эллиптических кривых над конечными полями в некоторых криптосхемах. Такая возможность уже рассматривалась ранее в работах $[21 ; 26]$.

В работе [21] для получения шифртекстов используется несколько эллиптических кривых, связанных между собой изогениями. При очередном шифровании случайным образом выбирают одну из них и с ее помощью получают шифртекст. Вместе с передачей шифртекста происходит передача и кривой, однако не той, с помощью которой проводилось шифрование, а некоторой другой, связанной с ней изогенией. Для преобразования кривых используют открытый и секретный ключи.

В работе [26] предложен метод построения двух изогенных кривых, одна из которых $E_{s}$ является секретной и обладает тем свойством, что атака GHS на ней достаточно эффективна. Вторая кривая $E_{p b}$ не является подверженной атаке GHS; она предоставляется пользователю и используется обычным образом в криптосхемах с открытым ключом. Первая кривая может 
быть впоследствии предъявлена генерирующей систему инстанцией некоторой проверяющей инстанции в качестве скрытого ключа. С ее помощью можно будет эффективно решать задачу дискретного логарифмирования на $E_{p b}$, в то время как без знания $E_{S}$ эта задача является трудной.

Ниже мы предлагаем еще несколько криптосхем, использующих изогенные кривые.

Первая схема представляет собой протокол электронного голосования. Мы предполагаем, что количество $r$ участвующих в голосовании невелико; такая схема может быть использована в каких-либо коммерческих волеизъявлениях. Проводящий голосование центр $A_{0}$ генерирует цепочку связанных изогениями кривых $E_{0}, E_{1}, \ldots, E_{r}$; мы обозначаем изогению, отображающую $E_{i}$ в $E_{i+1}$, через $\varphi_{i} ; \varphi_{r}$ есть такая изогения $E_{r}$ в $E_{0}$, что $\varphi_{r}=$ $=\varphi_{r-1} \bigcirc \ldots \circ \varphi_{0}$. Построение такой цепочки кривых можно осуществлять описанными в пп. 2, 3, 4 алгоритмами. Например, мы сначала выбираем кривую $E$ над конечным полем $\mathrm{GF}(q)$, затем вычисляем ее кондуктор, а потом для некоторых простых чисел, делящих кондуктор, строим изогении «вниз» или «вверх». После этого центр $A_{0}$ передает участнику голосования $A_{i}$ его секретную пару $E_{i}, \varphi_{i}$. Обозначим $l_{i}=\operatorname{deg} \varphi_{i}$. Тогда $\operatorname{deg} \varphi_{r}=$ $l_{r}=l_{0} \ldots l_{r-1}$.

Процесс голосования осуществляется следующим образом. Центр $A_{0}$ выбирает случайным образом точку $P$ на кривой $E_{0}$ и передает $A_{1}$ точку $P_{1}=\varphi_{0}\left(P_{0}\right), i$-й участник голосования передает $i+1$-му участнику точку $P_{i+1}=\varphi_{i}\left(2 P_{i}\right)$, если голосует «за», и точку $P_{i+1}=\varphi_{i}\left(3 P_{i}\right)$, если голосует «против» $\left(r\right.$-й участник передает точку центру $\left.A_{0}\right)$. В итоге центр $A_{0}$ получит точку $l_{r} 2^{k_{1}} 3^{k_{2}} P_{0}$, где $k_{1}-$ количество проголосовавших «за», $k_{2}-$ количество проголосовавших «против». Затем он определяет значения $k_{1}, k_{2}$ перебором (напомним, что $r$ невелико). Мы предполагаем здесь, что между $i$-м и $i+1$-м участниками существует секретный канал связи и что голосующие не нарушают правила голосования. При такой процедуре голосования нельзя определить, кто как проголосовал.

Аналогичную схему можно предложить для голосования с несколькими вариантами выбора, а не только «за» и «против». В такой схеме $i$-й голосующий, выбравший $j$-й вариант, умножает $P_{i}$ на $p_{j}$, где $p_{j}-j$-е простое число.

Теперь рассмотрим схему многоуровневой аутентификации. Предположим, что имеются множества пользователей $\mathbb{A}_{1}, \ldots, \mathbb{A}_{N}$, причем чем больше номер множества, тем более высокий приоритет имеют принадлежащие 
ему пользователи. Мы хотим, чтобы пользователь из множества $\mathbb{A}_{j}$ имел возможность аутентифицировать пользователя из множества $\mathbb{A}_{i}, i \leq j$.

Для решения этой задачи выберем достаточно большое конечное поле $\mathrm{GF}(q)$ и построим цепочку изогенных эллиптических кривых над этим полем $E_{0}, E_{1}, \ldots, E_{N}$; через $\varphi_{i}$ мы обозначаем изогению из $E_{i}$ в $E_{i+1}$. Кривые и изогении являются секретными, за исключением кривой $E_{0}$, которая считается общеизвестной. Мы считаем, что каждый пользователь имеет набор текущих идентификационных данных (включая, например, дату), которые можно представить стандартным образом в виде натурального числа. Пользователь из множества $\mathbb{A}_{i}$ должен знать все $E_{s}, \varphi_{s}, s \leq i$.

Процедура аутентификации пользователем из множества $\mathbb{A}_{j}$ пользователя из множества $\mathbb{A}_{i}, i \leq j$ заключается в следующем. Пользователь из множества $\mathbb{A}_{i}$ с идентификатором $m$ случайным образом выбирает точку $P_{0} \in E_{0}$ и вычисляет набор $P_{1}, \ldots, P_{i+1}$, где $P_{1}=\varphi_{0}\left(P_{0}\right), P_{2}=\varphi_{1}\left(P_{1}\right), \ldots, P_{i}=$ $=\varphi_{i-1}\left(P_{i-1}\right), P_{i+1}=\varphi_{i}\left(m P_{i}\right)$. Набор $P_{0}, P_{1}, \ldots, P_{i+1}$ он предъявляет пользователю из множества $\mathbb{A}_{j}$ в качестве аутентификационных данных. Тот проводит проверку очевидным образом, зная все необходимые кривые, изогении и идентификатор. Секретным ключом в такой схеме является набор кривых и изогений.

Подобная схема может быть использована в качестве электронной подписи к сообщению $m$.

Теперь покажем, как можно использовать изогенные кривые в криптосхемах, основанных на спариваниях Вейля и Тейта. Многочисленные примеры таких схем (без использования изогений) можно найти, например, в обзоре [8]. Мы будем пользоваться следующим свойством спаривания Вейля:

$$
e_{l}(P, \hat{\varphi}(Q))=e_{l}(\varphi(P), Q),
$$

где $\varphi: E_{1} \longrightarrow E_{2}-$ изогения эллиптических кривых $E_{1}, E_{2}$ над конечным полем $G F(q), P \in E_{1}[l], Q \in E_{2}[l]$ (см. [23]).

Предположим, что пользователь $A$ подписывает сообщение, направляемое к пользователю $B$, с помощью какой-либо схемы электронной подписи, основанной на спаривании Вейля и использующей общеизвестную эллиптическую кривую $E_{2}$. Он может вычислить и держать в секрете изогеную кривую $E_{1}$ и изогению $\varphi: E_{1} \longrightarrow E_{2}$, а вместе с подписью к сообщению, для вычисления которой использовалось спаривание точек $\varphi(P), Q$ на кривой $E_{2}$, передать также значение $\hat{\varphi}(Q) \in E_{1}$. Если затем в какой-то момент времени ему потребуется доказать, что именно он подписал сообщение, то он сможет предъявить кривую $E_{1}$ и точку $P$, такую, что $e_{l}(P, \hat{\varphi}(Q))=e_{l}(\varphi(P), Q)$. 
Восстановление кривой $E_{1}$ и точки $P$ по заданным $E_{2}, \varphi(P), Q, \hat{\varphi}(Q)$ является трудной задачей.

\section{7. Заключение}

Подводя итоги, скажем, что в данной работе мы описали эффективные алгоритмы построения изогений эллиптических кривых над конечными полями и их приложения в задаче дискретного логарифмирования в группах точек эллиптических кривых и в некоторых криптосхемах. Возможны и другие приложения изогений, например, к вычислению кратных точек на эллиптических кривых над конечными полями, см. [1], и к вычислению порядков групп точек эллиптических кривых, см. [3, гл. 7].

\section{Список литературы}

1. Василенко О.Н. Новые методы вычисления кратной точки на эллиптической кривой над конечным полем. - В сб.: Труды по дискретной математике. т. 11. - М.: ФИЗМАТЛИТ, 2008, с. 5-30.

2. Василенко О.Н. Теоретико-числовые алгоритмы в криптографии. - М.: МЦНМО, 2006, $334 \mathrm{c}$.

3. Blake I., Seroussi G., Smart N. Elliptic curves in cryptography. - Cambridge: Cambridge Univ. Press, 1999, 204 p.

4. Blake I., Seroussi G., Smart N. Advances in elliptic curve cryptography. - Cambridge: Cambridge Univ. Press, 2005, 296 p.

5. Bostan A., Morain F., Salvy B. Fast algorithms for computing isogenies between elliptic curves. - INRIA-00091441, version 1-6 Sep. 2006.

6. Cohen H. A course in computational algebraic number theory. - New York-BerlinHeidelberg: Springer-Verlag, 1993, $534 \mathrm{p}$.

7. Dewaghe R., Barua R., Sarkar P. Isogenie entre courbes elliptiques. - Utilitas Math., 1999 , v. 55 , p. $123-127$.

8. Dutta R., Barua R., Sarkar P. Pairing-based cryptographic protocols: a survey. Cryptology ePrint Archive, Report 2004/06.

9. Elkies $N$. Explicit isogenies. - Preprint, 1991.

10. Elkies $N$. Elliptic and modular curves over finite fields and related computational issues. - In: Computational perspectives on number theory: Proc. conf. honor of A. O. L. Atkin. - AMS, 1997, p. 21-76.

11. Enge A., Gaudry P. A general framework for subexponential discrete logarithm algorithms. - Acta Arithmetica, 2002, v. 102, № 1, p. 83-103.

12. Frey $G$. How to disguise an elliptic curve - Talk at Waterloo workshop on the ECDLP, 1998. http://cacr.math.uwaterloo.ca/conferences/1998/ecc98/slides.html

13. Galbraith S. D. Constructing isogenies between elliptic curves over finite fields. London Math. Soc. J. Comput. Math., 1999, v. 2, p. 118-138. 
14. Galbraith S. D., Hess F., Smart N. P. Extending the GHS Weil descent attack. - Lect. Notes Comp.Sci., 2002, v. 2332, p. 29-44.

15. Galbraith S.D., Smart N.P. A cryptographic application of Weil descent. - Lect. Notes Comp.Sci., 1999, v. 1746, p. 191-200.

16. Gaudry P., Hess F., Smart N. P. Constructive and destructive facets of Weil descent on elliptic curves. - J. Cryptology, 2002, v. 15, p. 19-46.

17. Hankerson D., Menezes A., Vanstone S. Guide to elliptic curve cryptography. New York-Berlin-Heidelberg: Springer-Verlag, 2004.

18. Jao D., Miller S.D., Venkatesan R. Ramanujan graphs and the random reducibility of discrete log on isogenous elliptic curves. - Cryptology ePrint Archive, Report 2004/312.

19. Kohel D. Endomorphism rings of elliptic curves over finite fields. - Berkeley: Univ. California, 1996.

20. Müller $V$. Ein Algorithmus zur Bestimmung der Punktzahl elliptischer Kurven über endlichen Körpern der Characteristik grősser drei. - Univ. Saarlandes, 1995.

21. Rostovtsev A., Stolbunov A Public-key cryptosystem based on isogenies. - Cryptology ePrint Archive, Report 2006/145.

22. Schoof $R$. Counting points on elliptic curves over finite fields - J. Theor. Nombr. Bordeaux, 1995, v. 7, p. 219-254.

23. Silverman J. The arithmetic of elliptic curves. - New York: Springer-Verlag, 1986.

24. Stewart I., Tall D. Algebraic number theory. - New York: Chapman and Hall, 1986.

25. Tate J. Endomorphisms of abelian varieties over finite fields. - Invent. Math, 1966, v. 2, p. 134-144.

26. Teske E. An elliptic curve trapdoor system. - J. Cryptolog., 2006, v. 19, № 1, p. $115-$ 133.

27. Vasilenko O.N. Number-theoretic algorithms in cryptography - Amer. Math. Soc. Transl. Math. Monogr. 2007, v. 232.

28. Vélu J. Isogénies entre courbes elliptiques - C.R.Acad. Sci. Paris, Sér. I., 1971, v. 273, p. 238-241. 